\title{
RESEARCH
}

Open Access

\section{Apelin enhances the osteogenic differentiation of human bone marrow mesenchymal stem cells partly through Wnt/ $\beta$-catenin signaling pathway}

Kai Hang ${ }^{1,2+}$, Chenyi $\mathrm{Ye}^{1,2+}$, Jianxiang X ${ }^{1,2+}$, Erman Chen ${ }^{1,2}$, Cong Wang ${ }^{1,2}$, Wei Zhang ${ }^{1,2}$, Lic Ni ${ }^{1,2}$, Zhih Kuang ${ }^{1,2}$, Li Ying ${ }^{1,2}$, Deting Xue ${ }^{1,2^{*}}$ and Zhijun Pan ${ }^{1,2^{*}}$ (D)

\begin{abstract}
Background: Management of fracture healing with a large bone defect remains a tricky subject in orthopedic trauma. Enhancing osteogenesis of human bone marrow-derived mesenchymal stem cells (hBMSCs) is one of the useful therapeutic strategies for fracture healing. Previous studies have revealed that Apelin may play an important role in bone metabolism. However, its function in the osteogenesis of hBMSCs remains unclear. Therefore, in this study, we investigated the effects and mechanism of Apelin on osteogenic differentiation.
\end{abstract}

Methods: We investigated the osteogenesis effects of hBMSCs by both exogenous Apelin protein and overexpression Apelin in vitro. Cell proliferation assay was used to assess the effect of Apelin on the proliferation of hBMSCs. ALP staining and Alizarin Red staining were used to evaluate ALP activity and mineral deposition respectively. QPCR and Western blotting analysis were used to detect the expression of target genes and proteins. In vivo, a rat tibial osteotomy model was established; radiographic analysis and histological evaluation were used to confirm the therapeutic effects of Apelin in fracture healing. Statistical significance was determined by two-tailed Student's $t$ test when 2 groups were compared. When more than 2 groups were compared, one-way ANOVA followed by Bonferroni's post-hoc test was used. And two-way ANOVA, followed by Bonferroni multiple comparisons post-hoc test, was performed when the treatment groups at different time points were compared.

Results: The addition of exogenous Apelin protein or overexpression of Apelin promoted osteoblast differentiation of hBMSCs in vitro. Increased mineral deposits were observed after treatment with extracellular Apelin protein or after the upregulation of Apelin. Moreover, $\beta$-catenin levels were upregulated by Apelin. The enhancement of osteogenic differentiation induced by Apelin was attenuated by specific Wnt/ $\beta$-catenin signaling pathway inhibitors. In a rat tibial osteotomy model, local injection of exogenous Apelin protein improved bone healing, as demonstrated by imaging and histological analyses.

Conclusions: Taken together, these findings indicate that Apelin regulates osteogenic differentiation of hMSCs partly via the $W n t / \beta$-catenin signaling pathway and effectively promotes fracture healing.

Keywords: Apelin, Osteogenesis, hBMSCs

\footnotetext{
* Correspondence: blueskine@zju.edu.cn; zrpzj@zju.edu.cn

${ }^{\dagger}$ Kai Hang, Chenyi Ye and Jianxiang Xu contributed equally to this work.

${ }^{1}$ Department of Orthopedic Surgery of the Second Affiliated Hospital, School

of Medicine, Zhejiang University, No. 88, Jiefang Road, Hangzhou 310009,

China

Full list of author information is available at the end of the article
}

(c) The Author(s). 2019 Open Access This article is distributed under the terms of the Creative Commons Attribution 4.0 International License (http://creativecommons.org/licenses/by/4.0/), which permits unrestricted use, distribution, and reproduction in any medium, provided you give appropriate credit to the original author(s) and the source, provide a link to the Creative Commons license, and indicate if changes were made. The Creative Commons Public Domain Dedication waiver (http://creativecommons.org/publicdomain/zero/1.0/) applies to the data made available in this article, unless otherwise stated. 


\section{Introduction}

Bone marrow-derived mesenchymal stem cells (BMSCs) are multipotent stromal cells that possess self-renewal capabilities and are able to differentiate into a variety of cell types, including osteoblasts, chondrocytes, adipocytes, and myocytes [1, 2]. Furthermore, BMSCs can be further induced to form tissues of mesodermal origin, including bones, cartilages, ligaments, muscles, tendons, and neurons [3]. The degree to which the culture will differentiate is determined by how the differentiation is induced, and varies among individuals [4]. Owing to these properties, BMSCs have been increasingly applied in regeneration medicine alone or in the form of a complex $[5,6]$.

The number of orthopedic trauma patients has been increasing along with the development of modern industries, and the treatment of patients with impaired fracture healing remains one of the most challenging clinical problems in trauma management [7]. There are two major fracture healing pathways-primary healing (also known as direct healing) and secondary healing (also known as indirect fracture healing). Secondary healing is the most common form of bone healing and consists of three major phases: (1) reactive phase, (2) reparative phase, and (3) remodeling phase [8]. After a fracture, acute inflammatory reactions release multiple initial inflammatory cytokines that dramatically enhance mesenchymal cell recruitment and osteogenesis $[9,10]$.

Apelin is the endogenous ligand for APJ, which is a seven trans-membrane $\mathrm{G}$ protein-coupled receptor [11] widely distributed in the limbs, heart, brain, adipose tissue, and kidney [12-14]. The Apelin/APJ system performs a broad range of activities in multiple organ systems. Apelin consists of numerous isoforms including Apelin-12, Apelin-13, Apelin-17, and Apelin-36, which are all derived from the C-terminal fragment of the Apelin pre-proprotein with 77 amino acids [15]. Among all these isoforms, Apelin-13 and its pyroglutamatemodified form Pyr-Apelin-13 are the most active isoforms that bind to the APJ receptor [16]. Apelin-13 exhibits protective effects in ischemic heart diseases, as well as anticonvulsive and neuroprotective properties. Meanwhile, Apelin-13 participates in the regulation of glucose homeostasis $[17,18]$. Previous studies have demonstrated that Apelin-13 stimulates human osteoblast proliferation and suppresses serum deprivation-induced apoptosis of MC3T3-E1 cells [19, 20].

In this study, we investigated the effects of exogenous Apelin-13 on osteogenesis differentiation of human bone marrow-derived mesenchymal stem cells (hBMSCs). We found that Apelin-13 enhanced osteogenic differentiation of hBMSCs through the $\mathrm{Wnt} / \beta$-catenin signaling pathway in vitro. We studied a rat tibial defect model treated with exogenous Apelin-13 by local injection and observed that Apelin-13 promoted healing of the bone defects in vivo.

\section{Materials and methods}

\section{Cell culture and reagents}

hBMSCs which can differentiate into osteoblasts, chondrocytes, and adipocytes under specific inductive conditions were purchased from Cyagen Biosciences (Guangzhou, China). Adherent hBMSCs were cultured in culture flasks with hMSC growth medium (Cyagen Biosciences, Guangzhou, China) at $37^{\circ} \mathrm{C}$ with $5 \% \mathrm{CO}_{2}$ in an incubator. hBMSCs were passaged when reached at $80-90 \%$ confluence. Cells from passages $3-8$ were used in subsequent experiments. Recombinant human Apelin-13(rhApelin-13) (Abcam, MA, USA) and recombinant DKK-1 (R\&D Systems) were purchased from Sino Biological. We used a DKK1 of $0.5 \mu \mathrm{g} / \mathrm{ml}$ based on a previous study [21].

\section{Cell proliferation assay}

To assess the effect of recombinant Apelin-13 and APELIN overexpression on the proliferation of hBMSCs, related cells were seeded into 96-well plate (5000/well). After $24 \mathrm{~h}$ adhering, the medium was changed by $10 \%$ Cell Counting Kit-8 (CCK-8, Dojindo, Kumamoto, Japan) in $100 \mu \mathrm{l}$ low-sugar Dulbecco's modified Eagle's medium (L-DMEM) for $4 \mathrm{~h}$ at $37^{\circ} \mathrm{C}$. Absorbance at 450 $\mathrm{nm}$, which is proportional to cell proliferation, was measured by a microplate reader (ELX808; BioTek, USA).

\section{Osteogenic differentiation protocol}

hBMSCs were cultured in growth medium (L-DMEM; $10 \%$ FBS (100-125, Gemini, USA) and $100 \mathrm{IU} / \mathrm{ml}$ penicillin/streptomycin) in 6- or 12-well cell culture plates at a density of $3 \times 10^{4} / \mathrm{cm}^{2}$ and incubated for $72 \mathrm{~h}$ at $37{ }^{\circ} \mathrm{C}$ with $5 \% \mathrm{CO}_{2}$. The cells were then cultured in osteogenic induction medium (L-DMEM with $10 \%$ FBS, $100 \mathrm{nM}$ dexamethasone, $100 \mathrm{IU} / \mathrm{ml}$ penicillin/streptomycin, 10 $\mathrm{mM} \beta$-glycerophosphate, and $0.2 \mathrm{mM}$ ascorbic acid). The cells were maintained by changing fresh osteogenic induction medium every $2-3$ days.

\section{ALP staining and ALP activity assay}

Cells were cultured in 12-well plates with osteogenic induction medium for 3 or 5 days. For ALP staining, cells were fixed with $4 \%$ paraformaldehyde for $20-30 \mathrm{~min}$. Cells were then washed by double distilled water $\left(\mathrm{ddH}_{2} \mathrm{O}\right)$ three times and stained by Alkaline Phosphatase Color Development Kit (Beyotime, Shanghai, China). To measure ALP activity, cells were lysed with lysis buffer consisted of 20 $\mathrm{mM}$ Tris- $\mathrm{HCl}$ (pH 7.5), 1\% Triton X-100, and $150 \mathrm{mM}$ $\mathrm{NaCl}$. ALP activity was determined by ALP activity assay (Beyotime). Finally, the conversion color of p-nitrophenyl phosphate was measured after 3 and 5 days of culture in an osteogenic medium at $405 / 650 \mathrm{~nm}$. 


\section{Alizarin Red staining}

Mineral deposition was assessed by Alizarin Red staining (ARS) (Cyagen Biosciences) after the induction of osteogenic differentiation. Cells were fixed with $4 \%$ paraformaldehyde for 20-30 min at room temperature and subsequently washed with $\mathrm{ddH}_{2} \mathrm{O}$ three times. The cells were incubated with a $0.2 \%$ solution of Alizarin Red for $5-10 \mathrm{~min}$ at room temperature, then rinsed with $\mathrm{ddH}_{2} \mathrm{O}$. The stain is incubated with $10 \%$ cetylpyridinium chloride (Sigma, Shanghai, China) for $1 \mathrm{~h}$ and collected the solutions, then $200 \mu \mathrm{l}$ was plated on a 96 -well plate and then was read at $560 \mathrm{~nm}$ by a microplate reader (ELX808; BioTek). The results were normalized to total protein concentration.

\section{RNA isolation and qPCR}

Total cellular RNA was isolated with RNAiso reagent (Takara, Dalian, China), and the solution was quantified by measuring the absorbance at $260 \mathrm{~nm}$ (NanoDrop 2000; Thermo Fisher Scientific). According to the manufacturer's instructions of the PrimeScript RT Master Mix (Takara), first-strand cDNA was synthesized. Total RNA $(\leq 1000 \mathrm{ng})$ was reverse-transcribed into cDNA using the Double-Strand cDNA Synthesis Kit (Takara) in a reaction volume of $20 \mu \mathrm{l}$. One microliter cDNA was used for qPCR. All gene transcripts were quantified by qPCR by SYBR Green PCR Master Mix (Takara) on ABI StepOnePlus System (Applied Biosystems, Warrington, UK). mRNAs of target genes and the housekeeping gene (GAPDH OR 18S) were quantified in separate tubes. All primers were synthesized by GENEray (Shanghai, China) . The cycle conditions were as follows: $95^{\circ} \mathrm{C}$ for $30 \mathrm{~s}$ then $95^{\circ} \mathrm{C}$ for $5 \mathrm{~s}$ for 40 cycles and $60^{\circ} \mathrm{C}$ for $30 \mathrm{~s}$. The relative target gene expression levels were calculated using the $2^{-\Delta \Delta \mathrm{Ct}}$ method.

\section{Western blotting analysis}

Cells were lysed in RIPA buffer combined with proteasome inhibitor and phosphatase inhibitors (Beyotime). Equal amounts of proteins were separated by $10 \%$ or $12 \%$ sodium dodecyl sulfate polyacrylamide gel electrophoresis, then separated target proteins were transferred to a polyvinylidene fluoride membrane (Millipore, Shanghai, China). After blocking in 5\% non-fat milk for $60 \mathrm{~min}$, the membranes were incubated overnight at $4{ }^{\circ} \mathrm{C}$ with antibodies specific to GAPDH (1:2000; Abcam, Shanghai, China), APELIN (1: 2000; Abcam, Shanghai, China), RUNX2 (1:1600; Abcam, Shanghai, China), COL1A1 (1:1000; Abcam, Shanghai, China), non-phosphorylated (active) $\beta$-catenin (1:1000; Abcam, Shanghai, China), or total $\beta$-catenin (1:1000; Abcam, Shanghai, China). After washing with TBST three times (10 min each), the membranes were incubated with secondary antibodies (horseradish peroxidase-conjugated, Beyotime) for $1 \mathrm{~h}$ at room temperature. After washing three times with TBST, proteins were detected using enhanced chemiluminescence blotting reagents (Millipore). Signal intensity was measured by Bio-Rad XRS chemiluminescence detection system (Bio-Rad, Hercules, CA, USA).

\section{Lentiviral packaging and cell infection}

Lentiviral overexpression APELIN (APELIN overexpression group (OE)) particles and lentiviral GFP particles, used as the control group (APELIN overexpression control group (OE-NC)), were prepared by Cyagen Biosciences (Guangzhou, China). Forty to 50\% confluent hBMSCs were incubated with lentiviral particles and $3 \mu \mathrm{g} / \mathrm{ml}$ polybrene in growth medium at a multiplicity of infection of 50 which is used as the optimized amount of virus determined by the GFP expression after lentiviral GFP particles infection. After $12 \mathrm{~h}$, the culture medium was changed. Three days later, transfected cells were passaged for use in subsequent experiments. The expression of APELIN was verified by qPCR and Western blotting analyses.

\section{Immunofluorescence analysis}

Cells were cultured in a 12-well plate with induction medium and evaluated for RUNX2 and APELIN using a fluorescence microscope (EU5888; Leica, Germany) as follows. Cells were fixed in $4 \%$ paraformaldehyde for 20 min at room temperature, permeabilized for $30 \mathrm{~min}$ in $0.2 \%$ Triton X-100, and blocked for $30 \mathrm{~min}$ in $2 \%$ bovine serum albumin. Fixed cells were washed and incubated overnight with anti-RUNX2 (1:1600; Abcam, Shanghai, China), APELIN (1:2000; Abcam, Shanghai, China), or COL1A1 (1:1000; Abcam, Shanghai, China). After washing three times with $\mathrm{ddH} 2 \mathrm{O}$, cells were incubated with a fluorescence-conjugated secondary antibody (Beyotime) for $1 \mathrm{~h}$, the nuclei were stained with 4',6-diamidino-2phenylindole (KeyGen Biotech, Nanjing, China) for 5 min. Target proteins were observed under a fluorescence microscope (Leica).

\section{In vivo evaluation in animals}

All Sprague-Dawley (SD) rats were purchased from the Academy of Zhejiang Province Medical Sciences. All animal experiments were in accordance with the Animal Care and Use Committee guidelines of Zhejiang University. All experimental procedures were in accordance with the Institutional Animal Care Use Committee at the second affiliated hospital of Zhejiang University (approve number: 2018-078).

In total, 18 male SD rats (8-week-old, approximately $200 \mathrm{~g}$ ) were used to establish rat tibial defect models. All rats were divided randomly and evenly into three groups: blank group, PBS (negative control group treated with PBS) group, and Apelin group ( $n=6$ per group). All surgical procedures were performed by two experienced orthopedic surgeons. The tibial defect model was 
established as reported previously [22, 23]. Rats were anesthetized intraperitoneally with $0.3 \%$ pentobarbital sodium (Sigma) at a dose of $30 \mathrm{mg} / \mathrm{kg}$ body weight. After anesthesia, an incision was made lateral to the tibia, away from the bone. A $1.3-\mathrm{mm}$ intramedullary fixation pin was inserted inside the medullary canal of the tibia for fixation. A 1.5-mm-diameter tibial defect was made in all SD rats approximately $7 \mathrm{~mm}$ from the proximal tibial growth plate by a hollow drill and punched through the cortex of the bone. The same leg was used for each group. Then rhApelin-13(50 $\mu \mathrm{g})$ was injected locally at the fracture site on days $0,3,5,8$, and 11 (i.e., immediately, $72 \mathrm{~h}$ ); PBS was used as a vehicle control. Animals were killed 6 weeks after surgery and collected the samples; then, samples were fixed in $4 \%$ paraformaldehyde for $72 \mathrm{~h}$ at room temperature for use in subsequent experiments.

\section{Radiographic analysis}

For the microcomputed tomography $(\mu \mathrm{CT})$ evaluation, tibia samples were scanned by a $\mu \mathrm{CT}-100$ imaging system (Scanco Medical, Brüttisellen, Switzerland) with X-ray energy settings of $70 \mathrm{kVp}, 1024$ reconstruction matrix, and $14.8 \mu \mathrm{m}$ slice thickness with an exposure time of $300 \mathrm{~ms}$. The $\mathrm{Tb} . \mathrm{N}$ and $\mathrm{BV} / \mathrm{TV}$ were evaluated by threedimensional standard microstructural analysis [24].

\section{Histological evaluation}

After $\mu \mathrm{CT}$ scanning, specimens ( $n=3$ for each group) were decalcified with $10 \%$ ethylene diaminetetra acetic acid (EDTA, Sigma) with $0.1 \mathrm{M}$ PBS for at least 2 months, with a solution change once a week. Thereafter, the specimens were embedded in paraffin in accordance with the standard procedures. Serial sections $(4 \mu \mathrm{m}$ thick) were cut and mounted onto polylysine-coated glass slides, deparaffinized, and then stained with HE, and Masson's trichrome was performed separately on consecutive tissue sections in accordance with our previous studies [25]. Finally, images were obtained on a traditional light microscopy (Leica DM4000B; Leica, Solms, Germany).

\section{Statistical analysis}

Statistical analysis was performed with SPSS 19.0 software (IBM, Armonk, NY, USA). All experiments were performed at least in triplicate, and the data are presented as means \pm SD. Statistical significance was determined by two-tailed Student's $t$ test when 2 groups were compared. When more than 2 groups were compared, one-way ANOVA followed by Bonferroni's post-hoc test was used. And two-way ANOVA, followed by Bonferroni multiple comparisons post-hoc test, was performed when the treatment groups at different time points were compared. A value of $P \leq 0.05$ was considered significant.

\section{Results}

Endogenous Apelin expression and the influence of exogenous recombinant Apelin-13 on proliferation and osteogenesis differentiation of $\mathrm{hBMSCs}$

To determine the expression level of Apelin associated with osteogenic differentiation of hBMSCs, we compared endogenous Apelin expression between undifferentiated and differentiated hBMSCs. In comparison with undifferentiated hBMSCs, mRNA and protein levels of Apelin showed no significant change after osteogenic differentiation on days $0,1,3$, and 5 (Fig. 1a-c). To determine whether exogenous Apelin-13 influences the proliferation of hBMSCs, we performed the CCK- 8 assay after the addition of different concentrations of Apelin$13(0-1000 \mathrm{nM})$ during days $0,1,3$, and 5 . No significant difference was detected in cell number among different concentrations of Apelin-13 (Fig. 1d). Then, we examined the effects of Apelin-13 on the differentiation of hBMSCs and found the expression level of specific osteogenesis-related genes, including collagen type I alpha 1 (COL1A1) and runt-related transcription factor 2 (RUNX2), increased dramatically after treatment with at least $0.1 \mathrm{nM}$ Apelin-13 on day 2. No obvious difference was observed after treatment with $1000 \mathrm{nM}$ Apelin-13 in comparison with $100 \mathrm{nM}$ Apelin-13 (Fig. 1e, f). COL1A1 and RUNX2 protein levels also increased significantly with at least $1 \mathrm{nM}$ Apelin-13 on day 2 (Fig. 1g-i). Afterwards, we used a concentration range of 0 to $100 \mathrm{nM}$ to study the effects of Apelin-13 on osteogenesis differentiation of hBMSCs.

\section{Apelin-13 increased the expression levels of osteo- specific genes and proteins and enhanced calcium deposit formation}

To assess the roles of Apelin-13 in osteogenic differentiation, we determined the expression levels of osteospecific genes and proteins including alkaline phosphatase (ALP), collagen type I alpha 1 chain (COL1A1), runt-related transcription factor 2 (RUNX2), and osteocalcin $(\mathrm{OCN})$ by Western blot and quantitative real-time PCR (qPCR) analyses.

Compared with the control group, Apelin-13 (ranging from 0 to $100 \mathrm{nM}$ ) significantly promoted the mRNA expression of COL1A1, RUNX2, and OCN during osteogenesis differentiation at days 1,3 , and 5 (Fig. 2a), whereas the mRNA expression of ALP markedly increased at days 1 and 3 (Additional file 1: Figure S2A). Furthermore, Western blot revealed that the protein levels of COL1A1 and RUNX2 were also remarkably higher following the increased mRNA expression at days 1, 3, and 5 (Fig. 2b).

We examined the calcium deposits that formed during late-stage osteogenic differentiation and maturation of osteoblasts by ARS. On days 9 and 11, Apelin-13 (0-100 


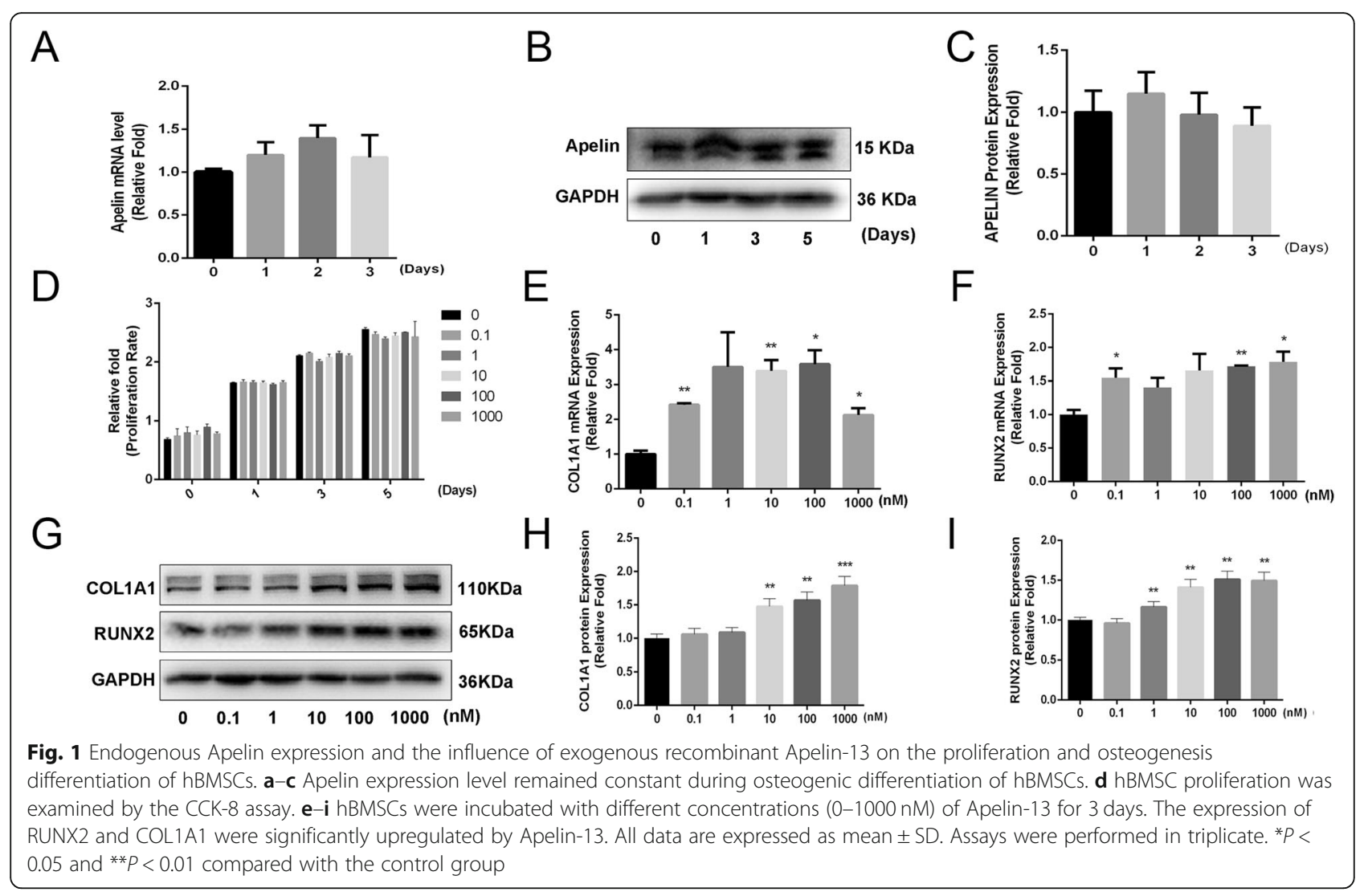

$\mathrm{nM}$ ) was found to considerably increase calcium deposits compared with the control group (Fig. 2c). Meanwhile, treatment with Apelin-13 during osteogenesis resulted in no significant difference of the ALP activity (Fig. 2d). Immunofluorescence (IF) assay revealed a significant increase in COL1A1 and RUNX2 protein expression after treatment with Apelin-13 (Additional file 2: Figure S2B).

\section{Apelin-13 promotes osteogenic differentiation of hBMSCs} partly via the $W n t / \beta$-catenin signaling pathway

To elucidate the specific signaling pathways through which Apelin-13 regulates hBMSC osteogenic differentiation, we used Western blot to examine the common signaling pathways involved in osteogenesis, including the PI3K/AKT signaling pathway, the MAPK signaling pathway, and the $\mathrm{Wnt} / \beta$-catenin pathway. The levels of total $\beta$-catenin and active $\beta$-catenin both increased after Apelin-13 (0-100 nM) treatment on day 3 of osteogenic differentiation. No significant changes were observed for the MAPK signaling pathway or the PI3K/AKT signaling pathway (Fig. 3a).

To further verify the involvement of Wnt $/ \beta$-catenin signaling pathway in the regulation of hBMSC osteogenic differentiation by Apelin-13, the Apelin-13-induced inhibitory effects of this pathway on osteogenesis were analyzed. After treatment with an appropriate concentration
(500 ng/ml) of DKK1, an effective inhibitor of the Wnt $/ \beta$ catenin signaling pathway, we found almost complete abrogation of the promotive effect on RUNX2 and COL1A1 expression induced by Apelin-13(100 nM) on day 3 (Fig. 3b).

Moreover, immunofluorescence assay revealed that the increases in RUNX2 and COL1A1 protein expression by Apelin-13(100 nM) were suppressed by DKK1 (500 ng/ ml) on day 3 (Fig. 3c).

\section{Endogenous Apelin overexpression increased the levels of osteo-specific proteins, enhanced ALP activity, and calcium deposit formation}

To clarify the roles of endogenous Apelin during osteogenic differentiation of hBMSCs, we constructed an Apelin overexpression hBMSC cell line through lentiviral vectors. Three days after infection and screening, Apelin expression level was determined by qPCR and Western blot analyses. In comparison with the control group, mRNA and protein levels of Apelin were significantly upregulated. Apelin protein expression was also assessed by immunofluorescence (IF) assay, and substantially higher Apelin expression level was observed in the Apelin overexpression group relative to the control group (Fig. 4a-d).

Western blot analysis showed higher expression levels of COL1A1 and RUNX2 proteins in the OE (APELIN 


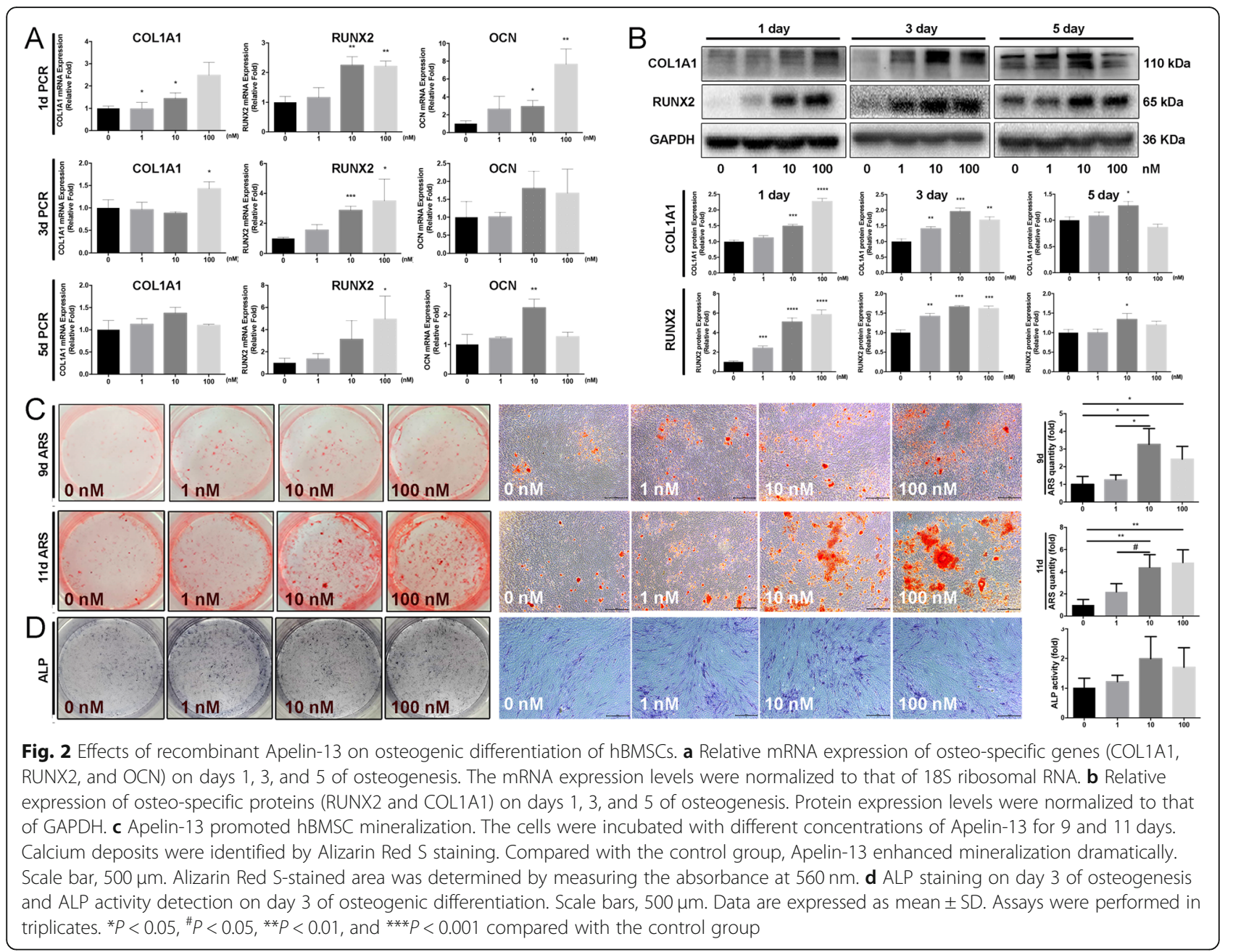

overexpression) group compared with the OE-NC (APELIN overexpression control) group on day 3 of osteogenic differentiation (Fig. 4e-g). Compared with the OE$\mathrm{NC}$ group, the activity of ALP increased in the OE group on day 3 of osteogenic differentiation (Fig. $4 \mathrm{~h}$, i). In addition, calcium mineralization was markedly enhanced in the OE group relative to the OE-NC group on day 9 (Fig. 4j, k).

\section{Addition of exogenous recombinant Apelin-13} accelerated bone healing in a rat tibial osteotomy model To prove the effects of Apelin-13 in vivo, exogenous Apelin-13 was administered in a rat tibial defect model. Microcomputed tomography $(\mu \mathrm{CT})$ analysis revealed that Apelin-13 promoted fracture healing (Fig. 5a-i). At 6 weeks of age, Apelin-13 significantly reduced the gap distance of cortical defect in comparison with the other two groups (Fig. $5 \mathrm{a}-\mathrm{C}$ ). The trabecular number and trabecular thickness were increased by Apelin-13 treatment (Fig. 5d, e). In addition to the trabecular phenotype, Apelin-13 also increased the thickness of the cortical bone (Fig. $5 \mathrm{f}-\mathrm{i}$ ).
Histological analysis demonstrated better cortex growth in the Apelin group compared with the other groups. (Fig. 5j, k).

\section{Discussion}

Successful osteogenesis of MSCs is essential for bone healing $[26,27]$. Herein, we provide the first evidence that Apelin may serve as a novel target for promoting the osteogenesis of hBMSCs.

Endogenous Apelin is constantly expressed in the osteogenesis process of hBMSCs, and previous studies have indicated that Apelin plays pivotal roles in bone metabolism. In accordance with our results, Apelin has been shown to suppress apoptosis in both BMSCs and the osteoblastic cell line MC3T3-E1, while exerts no effect on BMSC proliferation $[20,28]$. In hemodialysis (HD) patients with secondary hyperparathyroidism, higher PTH levels group had higher apelin levels $[1.17(0.7) \mathrm{ng} / \mathrm{ml}]$ compared with lower PTH levels group [0.50 (0.15) ng/ml], and Apelin protects human osteoblasts against apoptosis and induce osteoblast proliferation through competing with the opposite function of 


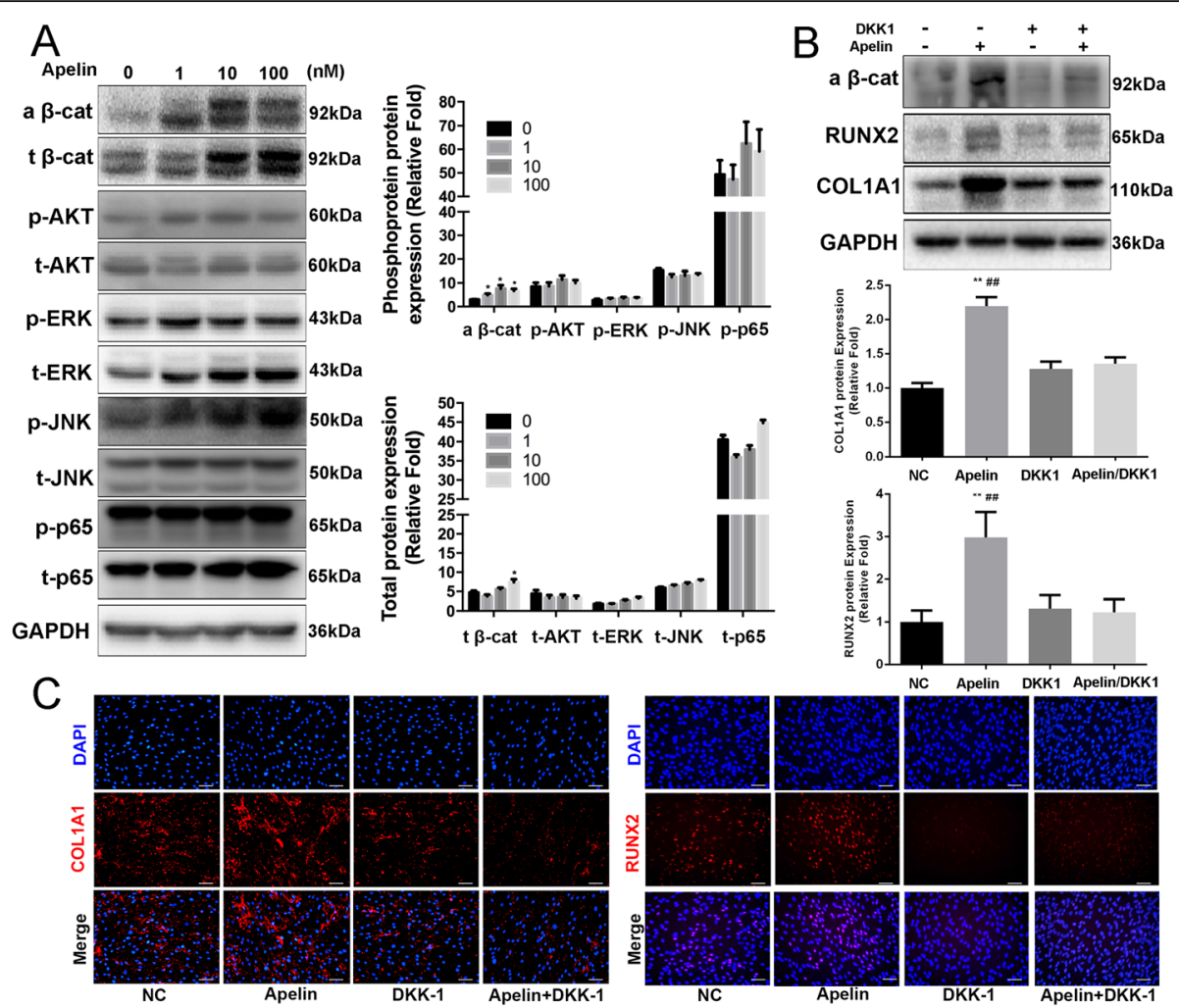

Fig. 3 Apelin-13 activated the Wnt/B-catenin signaling pathway. a Comparison of signaling pathway-related protein levels by Western blot analyses. hBMSCs were incubated with different concentrations of Apelin-13 during osteogenic differentiation on day 3. Protein expression levels were normalized to that of GAPDH. $\mathbf{b}$ Increased expression of osteo-specific proteins (COL1A1 and RUNX2) due to the inhibition of exogenous recombinant Apelin-13 by DKK1. Protein expression levels were normalized to that of GAPDH. c Immunofluorescence staining for RUNX2 and COL1A1. Scale bars, $100 \mu \mathrm{m}$. Data are expressed as mean \pm SD. Assays were performed in triplicate. ${ }^{*} P<0.05,{ }^{*} P<0.01$, and ${ }^{\# \#} P<0.01$, compared with the control group

PTH [29]. Apelin deficiency has been shown to contribute to the severe metabolic disturbance of the skeletal system [30, 31]. Our results revealed that Apelin-13 significantly upregulated the expression of osteogenic specific genes and proteins in hBMSCs. Meanwhile, ALP activity and mineral deposition were also enhanced by exogenous rhApelin-13 protein or overexpression of Apelin.

In Apelin-13-deficient mice, decreased expression of Wnt//-catenin signaling pathway-related molecules and downregulated collagen maturation-associated genes (loxl3 and loxl4) was observed [31]. Wnt comprises a large family of secreted glycoproteins that control cellular proliferation, differentiation, migration, apoptosis, survival, and polarity in various cell types [32]. Wnt proteins are known to play critical roles during skeletal patterning [33]. The Wnt receptors include 10 Frizzled family members, ROR2, Ryk, low-density lipoprotein receptor-related protein 5 (LRP5) and LRP6. Different Wnt proteins recognize their cognate receptors and activate at least three different intracellular signaling cascades: the canonical Wnt pathway (also known as the Wnt/ $\beta$-catenin pathway), the noncanonical Wnt pathway, and the Wnt-calcium pathway.
Wnt proteins activate the Frizzled/LRP5 or Frizzle/LRP6 receptor complexes and thus stabilize $\beta$-catenin in the cytoplasm. Subsequently, $\beta$-catenin is capable of entering the nucleus and regulaing the expression of Wnt target genes [34]. The canonical Wnt/ $\beta$-catenin signaling pathway has been shown to be closely associated with osteoblastogenesis [35-37] and to be one of the key regulators of skeletal lineage differentiation $[38,39]$. In our study, both the total $\beta$-catenin and active $\beta$ catenin expression levels increased dramatically in response to Apelin-13 during osteogenic differentiation. Moreover, the $\beta$-catenin-dependent Wnt signaling pathway inhibitor DKK1 almost completely abrogated the effects of Apelin-13 on osteogenesis. The results indicate that Apelin-13 promotes osteogenic differentiation of hBMSCs mainly through the Wnt/ $\beta$-catenin signaling pathway.

Lysyl oxidase genes (lox) including loxl3 and loxl4 are collagen maturation-associated genes, which are crucial to cross-link with collagen molecules through enzyme process [40]. What is the link between Apelin and lox genes expression needed further investigation. 


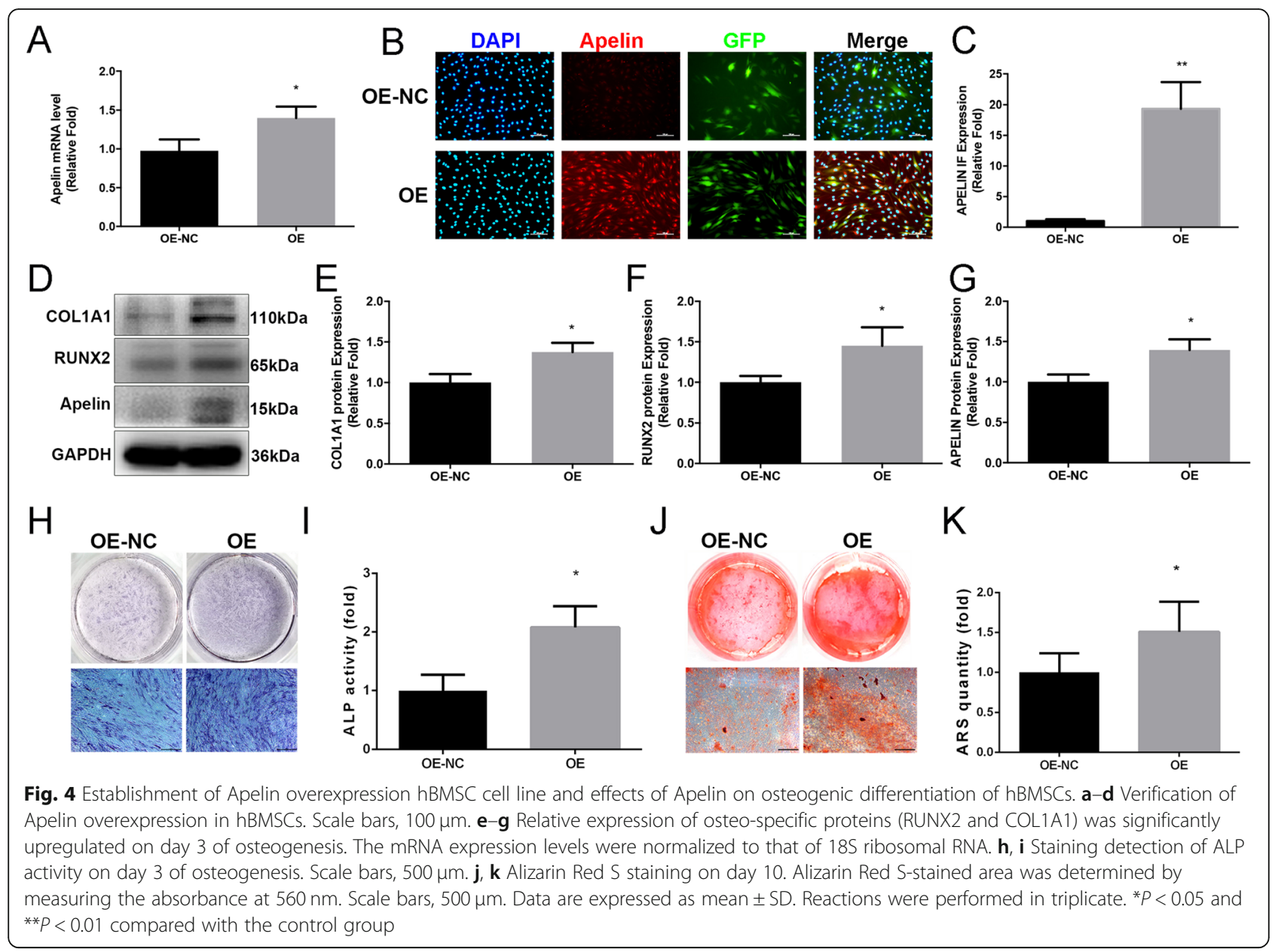

Previous studies have demonstrated that growth factors, such as bone morphogenetic proteins (BMPs), promote bone defect healing in vivo [41-45]. In our study, recombinant human Apelin-13 was shown to accelerate bone healing in a rat tibial osteotomy model.

However, our study has some limitations. First, although exogenous Apelin regulates the osteogenic differentiation of hBMSCs by activating $\mathrm{Wnt} / \beta$-catenin signaling pathway, we do not identify relevant cell surface receptor which mediates the transmission of signals. How Apelin/APJ system interacted with osteogenic signaling pathway needed a further study. Second, it remains unclear that whether accelerated osteogenic effect by endogenous overexpression Apelin through the same way as exogenous Apelin proteins. As mentioned above, the process of bone healing includes endochondral ossification and intramembranous ossification [27]. In this study, we have just investigated intramembranous ossification partially; however, the effects of the Apelin protein on the processes of endochondral ossification remains unclear. From the histological analysis, we can see better cortex growth treated with Apelin. However, a more quantitative analysis is needed to verify the new bone formation by Apelin. And because the small bone defect model used in our vivo study can heal spontaneously, the effect of Apelin is needed to be verified for larger bone defect model. At last, previous study has revealed that in normal healthy human subjects, the concentration of Apelin in plasma was $3.58 \pm 0.33 \mathrm{ng} / \mathrm{ml}$ [46]; unfortunately, it is unknown how plasma Apelin changed in bone fracture patients. Thus, we think further studies are needed.

\section{Conclusions}

Taken together, these findings indicate that Apelin regulates osteogenic differentiation of hMSCs partly via the $\mathrm{Wnt} / \beta$-catenin signaling pathway and effectively promotes fracture healing.

\section{Additional files}

Additional file 1: Figure S2A. The expression of ALP mRNA increased significantly on days 1 and 3 . Data are expressed as mean \pm SD. Assays were performed in triplicate. ${ }^{*} P<0.05$ compared with the control group. (TIF $46 \mathrm{~kb}$ ) 

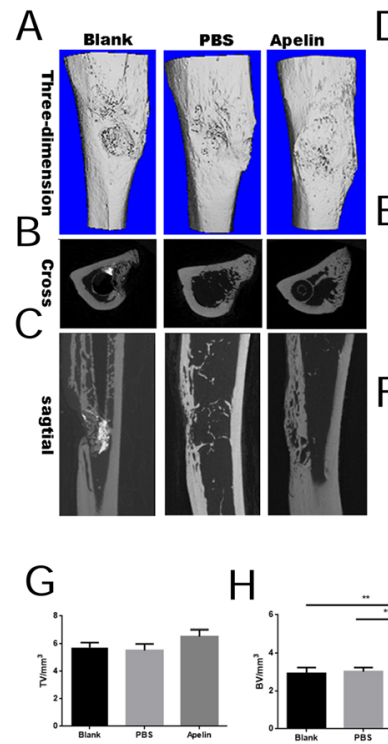

$\mathrm{H}$

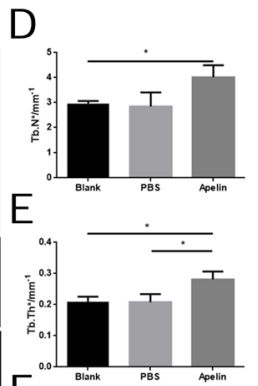

$\mathrm{F}$

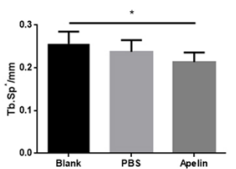

(gis

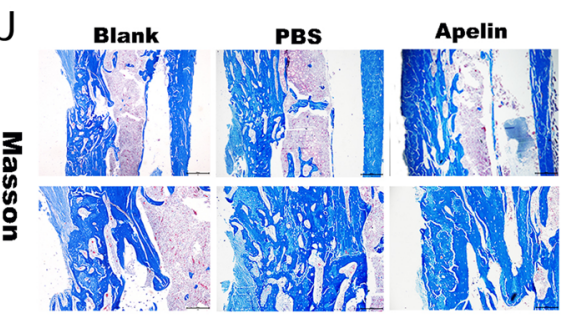

$\mathrm{K}$
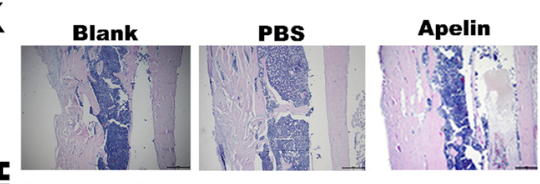

而
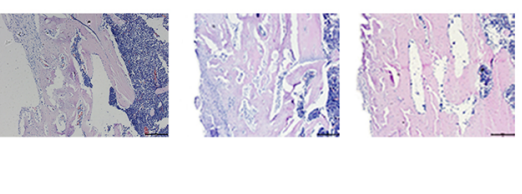

Fig. 5 Exogenous recombinant Apelin-13 accelerated bone healing in a rat tibial osteotomy model. a-c Microcomputed tomography analysis for bone healing. $\mathbf{d}-\mathbf{i}$ Bone volume and trabecular thickness were analyzed by microcomputed tomography. Data are expressed as mean \pm SD. Assays were performed in triplicate. ${ }^{*} P<0.05$ compared with the blank group. $\mathbf{j}$, k Histological analysis for bone healing. HE, hematoxylin and eosin staining; Masson, Masson's trichrome staining. Scale bars, $500 \mu \mathrm{m}$

Additional file 2: Figure S2B. Immunofluorescence staining of RUNX2 and COL1A1 proteins on day 3 of osteogenic differentiation (red). Cell nuclei were counter-stained with DAPI (blue). Scale bars, $100 \mu \mathrm{m}$. Data are expressed as mean $\pm \mathrm{SD}$. Assays were performed in triplicates. ${ }^{*} P<$ $0.05,{ }^{* *} P<0.01$ compared with the control group. (TIF $1198 \mathrm{~kb}$ )

\section{Abbreviations}

ALP: Alkaline phosphatase; ARS: Alizarin Red staining; CCK-8: Cell Counting Kit-8; COL1A1: Alpha-1 type I collagen; $\mathrm{dd}_{2} \mathrm{O}$ : Double distilled water; EDTA: Ethylene diaminetetra acetic acid; GAPDH: Glyceraldehyde-3phosphate dehydrogenase; hBMSCs: Human bone marrow-derived mesenchymal stem cells; HE: Hematoxylin and eosin; IF: Immunofluorescence; LDMEM: Low-sugar Dulbecco's modified Eagle's medium; LRP5: Lipoprotein receptor-related protein 5; MAPK: Mitogen-activated protein kinase; OCN: Osteocalcin; OE: Overexpression group; OE-NC: Overexpression control group; PI3K: Phosphoinositide 3-kinase; RUNX2: Runt-related transcription factor 2

\section{Acknowledgements}

We thank all clients from the Clinical Research Center of the Second Affiliated Hospital, Zhejiang University, including Xing Zhang, Jiayu Yang, Am Liu, and Chunc Li et al. We also appreciate the general help of LL Zhang from Orthopedic Research Center of the Second Affiliated Hospital, Zhejiang University.

\section{Authors' contributions}

DTX and ZP designed the research. $K H, C Y$, and EC performed the experiments. WZ, CW, JxX, LCN, ZhK, and LY analyzed the data. KH, DX, and $Z P$ wrote the paper. All authors read and approved the final manuscript.

\section{Funding}

This work was supported by grants from the National Natural Science Foundation of China (No. 81672147) and Zhejiang Provincial Natural Science Foundation of China (No. LQ19H060003).

\section{Availability of data and materials}

The datasets used and/or analyzed during the current study are available from the corresponding author on reasonable request.
Ethics approval and consent to participate

All animal experiments were in accordance with the Animal Care and Use Committee guidelines of Zhejiang University. All experimental procedures were in accordance with the Institutional Animal Care Use Committee at Zhejiang University.

\section{Consent for publication}

Not applicable.

\section{Competing interests}

The authors declare that they have no competing interests.

\section{Author details}

${ }^{1}$ Department of Orthopedic Surgery of the Second Affiliated Hospital, School of Medicine, Zhejiang University, No. 88, Jiefang Road, Hangzhou 310009,

China. ${ }^{2}$ Orthopedics Research Institute of Zhejiang University, No. 88, Jiefang Road, Hangzhou 310009, China.

Received: 27 January 2019 Revised: 22 May 2019

Accepted: 30 May 2019 Published online: 25 June 2019

\section{References}

1. Ankrum JA, Ong JF, Karp JM. Mesenchymal stem cells: immune evasive, not immune privileged. Nat Biotechnol. 2014;32(3):252-60.

2. Mahla RS. Stem cells applications in regenerative medicine and disease therapeutics. Int J Cell Biol. 2016;2016:6940283.

3. Dominici M, Le Blanc K, Mueller I, et al. Minimal criteria for defining multipotent mesenchymal stromal cells. The International Society for Cellular Therapy position statement. Cytotherapy. 2006;8(4):315-7.

4. Engler AJ, Sen S, Sweeney HL, Discher DE. Matrix elasticity directs stem cell lineage specification. Cell. 2006;126(4):677-89.

5. Park JS, Suryaprakash S, Lao YH, Leong KW. Engineering mesenchymal stem cells for regenerative medicine and drug delivery. Methods. 2015;84:3-16.

6. Wang $P$, Zhao L, Chen W, Liu X, Weir MD, Xu HH. Stem cells and calcium phosphate cement scaffolds for bone regeneration. J Dent Res. 2014;93(7):618-25.

7. Habal MB. Controlled bone regeneration: the ultimate process in bone repair. Clin Plast Surg. 2002:29(1):15-27 v.

8. Kalfas IH. Principles of bone healing. Neurosurg Focus. 2001;10(4):E1. 
9. Kon T, Cho TJ, Aizawa T, et al. Expression of osteoprotegerin, receptor activator of NF-kappaB ligand (osteoprotegerin ligand) and related proinflammatory cytokines during fracture healing. J Bone Miner Res. 2001; 16(6):1004-14

10. Einhorn TA, Majeska RJ, Rush EB, Levine PM, Horowitz MC. The expression of cytokine activity by fracture callus. J Bone Miner Res. 1995;10(8):1272-81.

11. O'Dowd BF, Heiber M, Chan A, et al. A human gene that shows identity with the gene encoding the angiotensin receptor is located on chromosome 11. Gene. 1993;136(1-2):355-60.

12. Blanchard A, Steichen O, De Mota N, et al. An abnormal apelin/vasopressin balance may contribute to water retention in patients with the syndrome of inappropriate antidiuretic hormone (SIADH) and heart failure. J Clin Endocrinol Metab. 2013;98(5):2084-9.

13. Wu L, Chen L, Li L. Apelin/APJ system: a novel promising therapy target for pathological angiogenesis. Clin Chim Acta. 2017;466:78-84.

14. De Falco M, De Luca $L$, Onori $N$, et al. Apelin expression in normal human tissues. In Vivo. 2002;16(5):333-6.

15. Tatemoto K, Hosoya M, Habata Y, et al. Isolation and characterization of a novel endogenous peptide ligand for the human APJ receptor. Biochem Biophys Res Commun. 1998;251(2):471-6.

16. Hosoya M, Kawamata Y, Fukusumi S, et al. Molecular and functional characteristics of APJ. Tissue distribution of mRNA and interaction with the endogenous ligand apelin. J Biol Chem. 2000;275(28):21061-7.

17. Chen D, Lee J, Gu X, Wei L, Yu SP. Intranasal delivery of Apelin-13 is neuroprotective and promotes angiogenesis after ischemic stroke in mice. ASN Neuro. 2015;7(5):1-15.

18. Kalantaripour TP, Esmaeili-Mahani S, Sheibani V, Asadi-Shekaari M, PasbanAliabadi $\mathrm{H}$. Anticonvulsant and neuroprotective effects of apelin-13 on pentylenetetrazole-induced seizures in male rats. Biomed Pharmacother. 2016;84:258-63.

19. Xie H, Tang SY, Cui RR, et al. Apelin and its receptor are expressed in human osteoblasts. Regul Pept. 2006;134(2-3):118-25.

20. Tang SY, Xie H, Yuan LQ, et al. Apelin stimulates proliferation and suppresses apoptosis of mouse osteoblastic cell line MC3T3-E1 via JNK and PI3-K/Akt signaling pathways. Peptides. 2007;28(3):708-18.

21. Yun HM, Park KR, Quang TH, et al. 2,4,5-Trimethoxyldalbergiquinol promotes osteoblastic differentiation and mineralization via the BMP and Wnt/betacatenin pathway. Cell Death Dis. 2015;6:e1819.

22. Kim SE, Yun YP, Shim KS, Kim HJ, Park K, Song HR. 3D printed alendronate-releasing poly (caprolactone) porous scaffolds enhance osteogenic differentiation and bone formation in rat tibial defects. Biomed Mater. 2016;11(5):055005.

23. Wei B, Huang C, Zhao M, et al. Effect of mesenchymal stem cells and platelet-rich plasma on the bone healing of ovariectomized rats. Stem Cells Int. 2016;2016:9458396

24. Bouxsein ML, Boyd SK, Christiansen BA, Guldberg RE, Jepsen KJ, Muller R. Guidelines for assessment of bone microstructure in rodents using microcomputed tomography. J Bone Miner Res. 2010;25(7):1468-86.

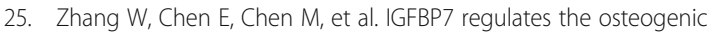
differentiation of bone marrow-derived mesenchymal stem cells via Wnt/ beta-catenin signaling pathway. FASEB J. 2018;32(4):2280-91.

26. Keramaris NC, Calori GM, Nikolaou VS, Schemitsch EH, Giannoudis PV. Fracture vascularity and bone healing: a systematic review of the role of VEGF. Injury. 2008;39(Suppl 2):S45-57.

27. Marsell R, Einhorn TA. The biology of fracture healing. Injury. 2011;42(6): 551-5.

28. Zeng X, Yu SP, Taylor T, Ogle M, Wei L. Protective effect of apelin on cultured rat bone marrow mesenchymal stem cells against apoptosis. Stem Cell Res. 2012:8(3):357-67.

29. Mafra D, Lobo JC, Farage NE, et al. The relationship between apelin and parathyroid hormone in hemodialysis patients. Ren Fail. 2012;34(8): 970-3.

30. Wattanachanya L, Lu WD, Kundu RK, et al. Increased bone mass in mice lacking the adipokine apelin. Endocrinology. 2013;154(6):2069-80.

31. Han XF, Zhang XX, Liu KM, Zhang Q. Apelin-13 deficiency alters cortical bone geometry, organic bone matrix, and inhibits Wnt/beta-catenin signaling. Gen Comp Endocrinol. 2018;267:29-35.

32. Kikuchi $A$, Yamamoto $H$, Sato A. Selective activation mechanisms of Wnt signaling pathways. Trends Cell Biol. 2009;19(3):119-29.

33. Manolagas SC. Wnt signaling and osteoporosis. Maturitas. 2014;78(3):233-7.
34. Clevers $\mathrm{H}$. Wnt/beta-catenin signaling in development and disease. Cell. 2006;127(3):469-80.

35. Bain G, Müller T, Wang X, Papkoff J. Activated $\beta$-catenin induces osteoblast differentiation of $\mathrm{C} 3 \mathrm{H} 10 \mathrm{~T} 1 / 2$ cells and participates in BMP2 mediated signal transduction. Biochem Biophys Res Commun. 2003;301(1):84-91.

36. Bodine PV, Zhao W, Kharode YP, et al. The Wnt antagonist secreted frizzledrelated protein-1 is a negative regulator of trabecular bone formation in adult mice. Mol Endocrinol. 2004;18(5):1222-37.

37. Hartmann C, Tabin CJ. Dual roles of Wnt signaling during chondrogenesis in the chicken limb. Development. 2000;127(14):3141-59.

38. Hill TP, Spater D, Taketo MM, Birchmeier W, Hartmann C. Canonical Wnt/ beta-catenin signaling prevents osteoblasts from differentiating into chondrocytes. Dev Cell. 2005;8(5):727-38.

39. Song L, Liu M, Ono N, Bringhurst FR, Kronenberg HM, Guo J. Loss of Wnt/ beta-catenin signaling causes cell fate shift of preosteoblasts from osteoblasts to adipocytes. J Bone Miner Res. 2012;27(11):2344-58.

40. Pacheco-Costa R, Kadakia JR, Atkinson EG, Wallace JM, Plotkin LI, Reginato RD. Connexin37 deficiency alters organic bone matrix, cortical bone geometry, and increases Wnt/B-catenin signaling. Bone. 2017;97:105-13.

41. Cowan CM, Soo C, Ting K, Wu B. Evolving concepts in bone tissue engineering. Curr Top Dev Biol. 2005;66:239-85.

42. Geiger $\mathrm{M}$, Li RH, Friess W. Collagen sponges for bone regeneration with rhBMP-2. Adv Drug Deliv Rev. 2003;55(12):1613-29.

43. Li RH, Wozney JM. Delivering on the promise of bone morphogenetic proteins. Trends Biotechnol. 2001;19(7):255-65.

44. Lutolf MP, Weber FE, Schmoekel HG, et al. Repair of bone defects using synthetic mimetics of collagenous extracellular matrices. Nat Biotechnol. 2003;21(5):513-8.

45. Skogh AC, Kihlstrom L, Neovius E, Persson C, Beckman MO, Engstrand T. Variation in calvarial bone healing capacity: a clinical study on the effects of BMP-2-hydrogel or bone autograft treatments at different cranial locations. J Craniofac Surg. 2013;24(2):339-43.

46. Chen Mary M, Ashley Euan A, Deng David XF, et al. Novel role for the potent endogenous inotrope Apelin in human cardiac dysfunction. Circulation. 2003;108(12):1432-9.

\section{Publisher's Note}

Springer Nature remains neutral with regard to jurisdictional claims in published maps and institutional affiliations.

Ready to submit your research? Choose BMC and benefit from:

- fast, convenient online submission

- thorough peer review by experienced researchers in your field

- rapid publication on acceptance

- support for research data, including large and complex data types

- gold Open Access which fosters wider collaboration and increased citations

- maximum visibility for your research: over $100 \mathrm{M}$ website views per year

At BMC, research is always in progress.

Learn more biomedcentral.com/submissions 